\title{
An Innovation in Practice for the Agency for Healthcare Research and Quality (AHRQ): Initiative for a Comprehensive Foot Examination across Health Care Settings Diagnostics and Treatment
}

\author{
Michele Burdette Taylor* \\ Assistant Professor, Community Health, ANCC Board Certified in Professional Development WOCN Board Certified in Foot and Wound Care, USA
}

Submission: September 13, 2018 Published: September 20, 2018

*Corresponding author: Michele Burdette Taylor, Assistant Professor, Community Health, ANCC Board Certified in Professional Development WOCN Board Certified in Foot and Wound Care, USA, Tel: 907-330-9928; Email: mrburdettetaylor@alaska.edu

\section{Opinion}

Diabetes and heart disease have now superseded cancer deaths in the United States of America. Disease, disability, endof-life care, and co-morbidities are expensive and devastating. Utilizing a Certified Foot Care Nurse (CFCN) has tremendous potential for meeting the challenge of reducing wounds leading to amputations. Our national goal is to improve health care, remove barriers, and focus on the aging obese Medicare population in efforts to reduce costs and improve care. Medicare programs focus on preventative care where, Certified Foot and Nail Care Nurse (CFCN) may assume a key role in reducing the costly impact of care for people with diabetes, lower extremity arterial disease, and osteoarthritis. Since 1993, Medicare has provided coverage for a pair of shoes and three sets of inserts annually. Since 2006 Medicare took the first step towards "pay for performance" reimbursements, a voluntary program for reporting data about care delivered and referrals for the Therapeutic Shoe Bill. CFCN's may be the key proactive preventive partner to reduce ulceration, facilitate patients' footwear fitting and use, and implement use of over-the-counter compression socks.

Nurses' are primed for increasing preventive information and knowledge for Medicare beneficiaries and appropriate and timely referrals for early intervention and saving Medicare money. This initiative for innovation in practice is in direct response to the Institute of Medicine (IOM) and the Agency for Health Care Quality Research (AHRQ). The AHRQ and IOM have charged nurses to practice to the full extent of their education and training and develop innovative health care proposals to specifically to lead change, improve care, and reduce costs. The significance of this innovative idea is that reimbursement should reward interventions that are therapeutic and reduce utilization. The estimated cost of a foot ulcer is $\$ 4,595$ per episode to nearly $\$ 28,000$ per Medicare Beneficiary with national cost of $\$ 5$ billion. The estimated cost of a Lower Extremity Amputation (LEA) is $\$ 30,000$ per event with total national cost of $\$ 1.6$ billion, with the added costs of prior ulcer care costs exceed $\$ 6$ billion annually.
The evidence supports the fact that the primary and secondary interventions to prevent a foot ulcer, is a comprehensive foot exam and daily use of therapeutic shoes. Nurses are the leader in health care for proactive prevention. Education and referral to prevent hospital readmissions, by focusing on those with Lower Extremity Neuropathic Disease (LEND) and Lower Extremity Arterial Disease (LEAD) is complicated, but by identifying patients at risk and qualifying the degree of risk, while monitoring the HbA1c for tight glucose control may reduce the number of non-traumatic amputations in the USA. Falls due to LEND and LEAD is also very serious, so monitoring the beneficiaries is also important. With a comprehensive foot exam of patients admitted in any setting, determining loss of protective sensation (LOPS) and compromised blood flow allows for early detection, treatment protocol implementation, education, and referral. Certified Foot Care Nurses (CFCN) by their training and certification are responsible for conducting a comprehensive lower extremity assessment, providing education, surveillance, and referral so patients are aware of actual or potential complications and encouraged to inspect their feet daily. Nurses' report and facilitate care at the first sign of a complication (no matter how minor) to prevent problems developing into more serious and expensive issues. A comprehensive foot exam can be done in 10-15 minutes, focusing initially on loss of protective sensation (LOPS) and lower extremity arterial disease (LEAD). At the same time, Medicare beneficiaries are educated about foot care, daily needs, and problems to report. The benefits of utilizing a Certified Foot Care Nurse (CFCN) are numerous. With a high population of aging people with diabetes, heart disease, and obesity, utilizing a nurse to assist with management of foot issues improves the chances of reducing a lower extremity wound and subsequent amputation. It is prudent to structure care in any setting to incorporate foot care at a basic level intervention.

Foot care nurses are monitoring their clientele every 8 weeks routinely, as a service for nail debriding, reducing corns and 
calluses, and monitoring blood values and foot wear. The major aspects of most benefit are if the CFCN is utilized as a member of the multidisciplinary team. The IOM also insists that nurses be full partners in the care of the Medicare beneficiary. By conducting a comprehensive foot exam, directing care and attention to any wounds, injuries, or infections, while serving as triage is crucial to the overall goal of addressing needs efficiently and costeffectively. Education, repeat education, and reinforcement utilizing motivational strategies and a variety of teaching methods allows for greater dissemination and acquisition of knowledge.

The CFCN conducts a tactile and vibratory sensation examination on every Medicare beneficiary using a 5.07 / 10 gram monofilament and $128 \mathrm{mHZ}$ tuning fork. Detecting neuropathic changes and instituting corrective therapy and safety precautions such as medicine management, monitoring $\mathrm{HbA1c}$, gait analysis, and pain management helps prevent the initial injury of the lower extremity. Conducting the non-invasive vascular assessment to include Doppler pulses, performing an Ankle-Brachial Index (ABI), and Toe Pressures (TP) identified those with vascular compromise and possible future cardiovascular insult.

Lower extremity arterial disease is common with people with diabetes, those over 70 years of age, and/or those between 5570 years of age who have a history of smoking. Appropriate and immediate referral to a vascular specialist is considered early intervention, in the prevention of amputation. Palliative care and triage are also critical to limb preservation and promotion of comfort for sustaining quality of function and safety. Mobilizing the CFCN can be crucial to early detection, education, and referral. By assessing any signs of infection, dermatologic, musculoskeletal, neuropathic, and vascular status, patients can identified and fasttracked to the appropriate intervention and specialty. Education has always been the best method of wound and amputation prevention. Nurses, as the most trusted health care provider, are a natural fit for people with diabetes and the older population. Trusted communication, rapport, and sustaining relationships for the long - term allows for transferring of knowledge while demonstrating care, compassion, and competency. Providing information in a variety of formats, using adult learning principles, and incorporating a weekly phone call to check on the beneficiary is interpreted as caring and assists in ensuring cooperation among patients and caregivers.

Utilizing the Wound Ostomy Continence Nurses' Credentialing Board (WOCNCB) Certified Foot and Nail Care Nurse (CFCN) raises the standard of care substantially and reduces overall costs to life, limbs and Medicare dollars. This innovation in practice to improve health care delivery includes, diagnostics and intervention that focuses on early detection of LEND and LEAD and intervention by, initially conducting a 10-15minute comprehensive foot exam. This proposal is a simple innovation in diagnostics and treatment that could have tremendous positive outcomes nationwide.

\section{Your next submission with Juniper Publishers will reach you the below assets}

- Quality Editorial service

- Swift Peer Review

- Reprints availability

- E-prints Service

- Manuscript Podcast for convenient understanding

- Global attainment for your research

- Manuscript accessibility in different formats

( Pdf, E-pub, Full Text, Audio)

- Unceasing customer service

Track the below URL for one-step submission https://juniperpublishers.com/online-submission.php 